\title{
Photoluminescence properties of GaN grown on compliant silicon-on-insulator substrates
}

\author{
J. Cao, D. Pavlidis, ${ }^{\text {a) }}$ and A. Eisenbach \\ Solid State Electronics Laboratory, Department of Electrical Engineering and Computer Science, \\ The University of Michigan, Ann Arbor, Michigan 48109-2122
}

\author{
A. Philippe, C. Bru-Chevallier, and G. Guillot \\ Laboratoire de Physique de la Matière, Institut National des Sciences Appliquées de Lyon, \\ CNRS UMR5511, Bâtiment 502, 20, 69621 Villeurbanne Cedex, France
}

(Received 15 August 1997; accepted for publication 3 October 1997)

\begin{abstract}
A compliant substrate approach has been employed to release lattice-mismatch caused strain in GaN epilayers through stress absorption in the substrate. GaN layers have been grown on silicon-on-insulator (SOI) substrates by low-pressure metalorganic chemical vapor deposition. Photoluminescence measurements at $4 \mathrm{~K}$ show the spectrum of grown GaN being dominated by UV emission around $3.47 \mathrm{eV}$ related to neutral-donor bound excitons. The much weaker yellow luminescence shows a broad spectrum around $2.16 \mathrm{eV}$. Peak position of the UV emission changes both with measurement temperature and strain. At room temperature, the UV peak is red shifted by $64 \mathrm{meV}$ corresponding well to the band-gap temperature dependence. Strain-induced blue shift of the peak, compared to unstrained GaN, is much less than for growth on sapphire, indicating strain relief in the GaN by growth on SOI. Further reduction of the blue shift is consistent with increased electron mobility. (C) 1997 American Institute of Physics. [S0003-6951(97)03348-2]
\end{abstract}

Extensive research efforts have been devoted to obtain high quality $\mathrm{GaN}$ and related nitrides because of their promise in (UV)/blue light emitters ${ }^{1}$ and high-power, hightemperature electronic devices. ${ }^{2}$ Improvement of material quality has been hindered by the lack of an ideal substrate that is well latticed and thermally matched. The mismatch leads to high interfacial strain between epilayer and substrate and causes a large defect density in the GaN epitaxial layers.

To release epilayer strain, compliant substrates have been proposed for lattice-mismatched epitaxy. ${ }^{3-7}$ Thick, lattice-mismatched InGaAs epitaxial layers have been grown on compliant GaAs substrates via molecular beam epitaxy (MBE). ${ }^{3,4}$ Most recently, thick dislocation-free InGaP layers have been grown on twist-wafer-bonded GaAs compliant substrates. ${ }^{5}$ Various types of compliant substrates can be envisaged but techniques that offer the potential of large wafer size are obviously most attractive since they are best suited for device and circuit applications. The compliant substrate reported by the authors in the past ${ }^{6,7}$ addresses exactly this need by employing as substrate the thin Si overlay of the silicon-on-insulator configuration in order to accomodate most of the strain. Silicon-on-insulator (SOI) substrates offer also the possibility of $\mathrm{GaN}$ device incorporation into siliconbased integrated circuits in order to perform more complex functions.

The use of SOI substrates has been reported for (MBE) growth of SiGe. ${ }^{8}$ Also, GaN was grown on the SiC-SOI heterostructure using $\mathrm{MBE}^{9,10}$ in order to exploit the advantage of this compliant substrate. Improved results for GaN layers grown by metalorganic chemical vapor deposition (MOCVD) directly on SOI substrates without previous formation of $\mathrm{SiC}$ have been reported by the authors. 6,7

According to the compliant substrate principle, the strain

${ }^{\text {a)} E l e c t r o n i c ~ m a i l: ~ p a v l i d i s @ u m i c h . e d u ~}$ in a lattice-mismatched epilayer can be reduced, with respect to growth on a thick $\mathrm{Si}$ or sapphire substrate, via partial accommodation of the total strain in a thin compliant overlay. ${ }^{11,12}$

The SOI substrates used in this work were fabricated by SIMOX (separation by implanted oxygen) technology. ${ }^{13}$ Thicknesses of silicon overlay and $\mathrm{SiO}_{2}$ buried layer were $52.8 \pm 1.1 \mathrm{~nm}$ and $81.2 \pm 4.4 \mathrm{~nm}$, respectively. The Si substrate was (100)-oriented. GaN layers were grown on SOI substrates in a modified EMCORE GS-3200 low-pressure (60 Torr) MOCVD system, using $\mathrm{H}_{2}$ as carrier gas (3000 sccm). Trimethylgallium (TMGa) and Ammonia $\left(\mathrm{NH}_{3}\right)$ were used as $\mathrm{Ga}$ and $\mathrm{N}$ precursors, respectively. The $\mathrm{NH}_{3}$ flow rate was constant $2000 \mathrm{sccm}$ both during buffer and bulk $\mathrm{GaN}$ growth. The TMGa flow rate was $36 \mu \mathrm{mol} / \mathrm{min}$ during bulk growth $(\mathrm{V} / \mathrm{III}=2500$, growth rate $\sim 0.8 \mu \mathrm{m} / \mathrm{h})$ and 18 $\mu \mathrm{mol} / \mathrm{min}$ during buffer growth. Thermal annealing/ nitridation temperature prior to GaN buffer growth $(\sim 250 \AA$ thick) was $1040{ }^{\circ} \mathrm{C}$, buffer growth temperature was $500{ }^{\circ} \mathrm{C}$, and bulk growth temperature was $940{ }^{\circ} \mathrm{C}$.

The quality of the grown $\mathrm{GaN}$ layers was examined by standard single crystal $(\theta-2 \theta)$ x-ray diffraction (XRD), scanning electron microscopy (SEM), van-der-Pauw Hall measurements, and photoluminescence (PL) measurements. The PL laser was an argon laser by COHERENT equipped with an intra-cavity prism to select the $334 \mathrm{~nm}$ emission line from its spectrum. The output power was constant $15 \mathrm{~mW}$.

Compared with GaN layers grown directly on $\mathrm{Si}$ substrates, crystal uniformity, surface morphology, and number of threading dislocations of GaN layers grown on SOI substrates are improved as evidenced by XRD and transmission electron microscopy (TEM). ${ }^{6}$ Full width at half maximum (FWHM) XRD values improved from 672 to 378 arc sec by growth on SOI instead of $\mathrm{Si}$ substrates. ${ }^{6}$ The GaN layers grown directly on $\mathrm{Si}$ substrates are highly resistive while 


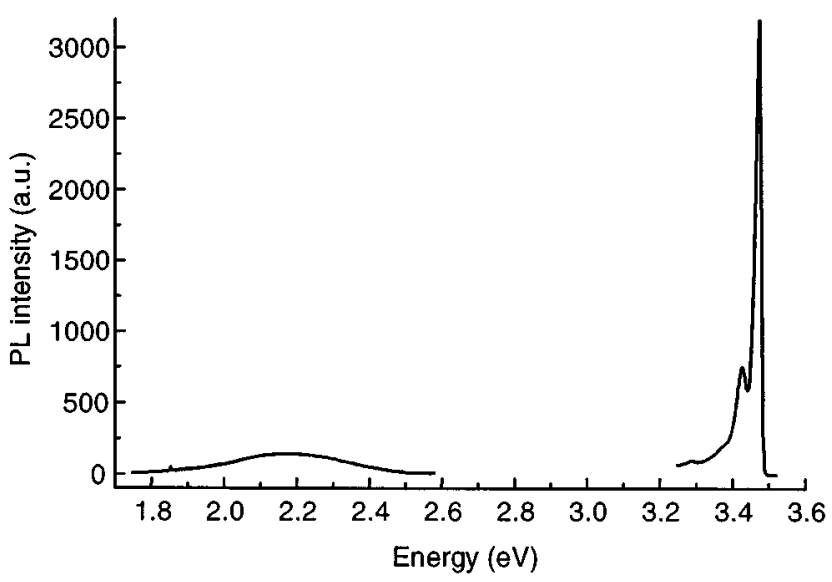

FIG. 1. PL spectrum at $6 \mathrm{~K}$ of a $1.2 \mu \mathrm{m}$ thick GaN layer grown on SOI (sample A).

those on SOI substrates are unintentionally $n$ type. Crystal quality, surface morphology, and especially electron mobility of GaN on SOI improve by increasing the growth temperature, the buffer growth rate, and by reducing the thickness of the top Si layer of the SOI substrate. The latter results in a FWHM reduction of the GaN (0002) XRD peak from 510 to 366 arc sec, and an electron mobility increase by an order of magnitude to $350 \mathrm{~cm}^{2} / \mathrm{Vs}$ at a low background carrier concentration of $1.8 \times 10^{17} \mathrm{~cm}^{-3}$.

PL measurements have been performed to evaluate the optical properties of nominally undoped, $1.2 \mu$ m-thick GaN layers grown on SOI substrates. A representative PL spectrum (sample A) taken at $6 \mathrm{~K}$ is shown in Fig. 1. The electron mobility and the background carrier concentration of this sample are $320 \mathrm{~cm}^{2} / \mathrm{Vs}$ and $3.9 \times 10^{17} \mathrm{~cm}^{-3}$, respectively. The dominant photoluminescence at low temperature is the near-bandgap emission around $3.47 \mathrm{eV}$ attributed to the neutral-donor bound exciton. ${ }^{14}$ The other major feature of the luminescence spectrum is a very broad, low energy luminescence band centered at about $2.16 \mathrm{eV}$ with an FWHM of $360 \mathrm{meV}$, commonly referred to as yellow luminescence. The UV/yellow luminescence peak intensity ratio is 23 at $6 \mathrm{~K}$.

At room temperature (RT), the near-bandgap emission is still detectable, indicating good quality of the GaN epilayers grown on SOI. The peak intensity of the UV emission at RT is reduced compared to $6 \mathrm{~K}$. This is generally observed and due to nonradiative processes. The decreasing UV/yellow luminescence peak intensity with increased temperature was also reported elsewhere. ${ }^{14}$ The yellow luminescence has a broad spectrum even at low temperatures. As the measurement temperature is increased from 6-300 K, peak position and FWHM remain almost constant, indicating that the yellow luminescence is associated with widely spread energy levels deep within the band gap.

The above described mechanism for the yellow luminescence which involves deep $\mathrm{C}$ acceptors is consistent with our investigation of the composition of the epitaxial layers. Xray photoelectron spectroscopy (XPS) and secondary ion mass spectroscopy (SIMS) analysis of GaN grown on SOI reveal the presence of $\mathrm{O}, \mathrm{H}, \mathrm{Si}$, and $\mathrm{C}$ impurities in the

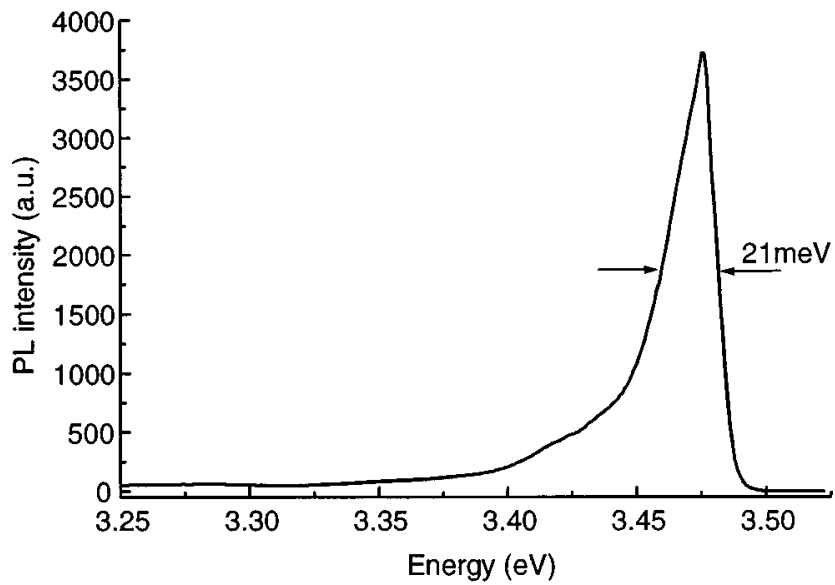

FIG. 2. UV luminescence of GaN on SOI (sample B, grown at $\sim 0.8 \mu \mathrm{m} / \mathrm{h}$ and $\mathrm{V} / \mathrm{III}=2500$ ) measured at $6 \mathrm{~K}$.

epilayer, which may also contribute to the background carrier concentration. The $\mathrm{C}$ concentration in the $\mathrm{GaN}$ epilayer is in the order of $10^{18}-10^{19} \mathrm{~cm}^{-3}$, which may well contribute to the yellow luminescence. The source of $\mathrm{C}$ incorporated in the $\mathrm{GaN}$ epilayers is not clear at this point. It may come from the TMGa, the graphite susceptor or its $\mathrm{SiC}$ coating, both of which may release $\mathrm{C}$ atoms into the chamber during high temperature growth. For optoelectronic applications of $\mathrm{GaN}$ in short wavelength range, the competition between yellow and UV luminescence will decrease the efficiency of the devices, especially for devices at room temperature. From the above discussion, decreasing the carbon incorporation in the epitaxial layer may help to reduce the yellow luminescence of $\mathrm{GaN}$ and improve crystal quality.

Figure 2 shows the details of the UV emission PL spectrum at $6 \mathrm{~K}$ of another sample of GaN grown on SOI (sample $\mathrm{B}$, electron mobility and background carrier concentration of this sample are $178 \mathrm{~cm}^{2} / \mathrm{Vs}$ and $2.8 \times 10^{17} \mathrm{~cm}^{-3}$, respectively). At low temperature, the peak is centered at $3.473 \mathrm{eV}$ and the FWHM is a moderately low $21 \mathrm{meV}$, indicating reasonable good quality of the GaN epilayer.

At room temperature, the UV luminescence peak intensity is decreased due to nonradiative transitions and its FWHM is considerably larger. Compared to the spectrum at low temperature, the near band-gap emission is shifted to lower energies. This experimental $63 \mathrm{meV}$ red shift of (from $3.473 \mathrm{eV}$ at $6 \mathrm{~K}$ to $3.410 \mathrm{eV}$ at $300 \mathrm{~K}$ ) is in excellent agreement with the theoretical $66 \mathrm{meV}$ shift due to the temperature dependence of the $\mathrm{GaN}$ band gap which is given by ${ }^{16}$

$$
E_{g}(T)=3.503 \mathrm{eV}-\frac{5.08 \times 10^{-4}(\mathrm{eV} / \mathrm{K}) T^{2}}{996 \mathrm{~K}-T} .
$$

For undoped $\mathrm{GaN}$, the position of the neutral-donor bound exciton peak at low temperatures can also reveal the strain existing in the GaN epilayer. Detailed PL studies have been reported on relatively thick $(50-100 \mu \mathrm{m}) \mathrm{GaN}$ samples grown by chloride vapor transport method on (0001) sapphire substrates, where the neutral-donor bound exciton peak is found at $3.469 \mathrm{eV}$. Because of the layer thickness, it seems reasonable to assume that the $\mathrm{GaN}$ epilayer is completely relaxed and the above emission energy should be character- 


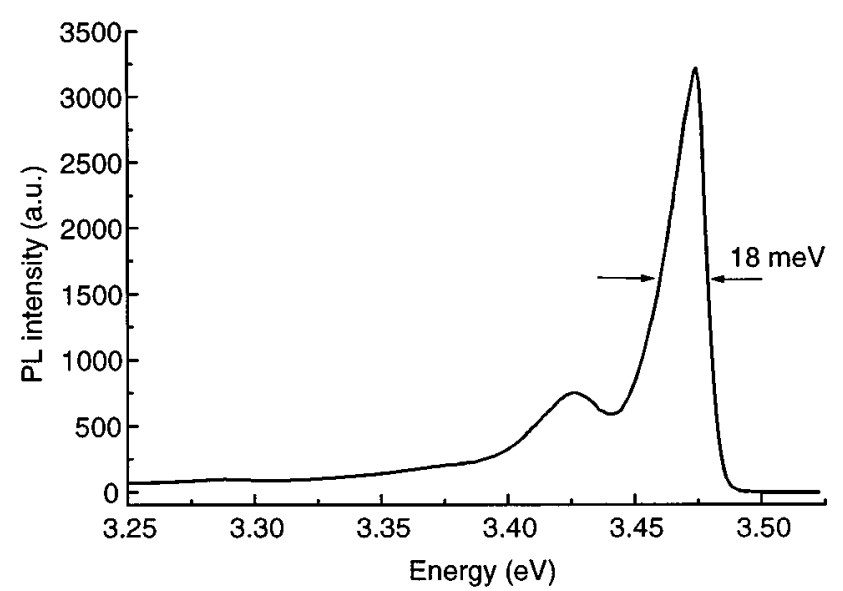

FIG. 3. UV luminescence of GaN on SOI (sample A, grown at $\sim 1.5 \mu \mathrm{m} / \mathrm{h}$ and $\mathrm{V} / \mathrm{III}=3000$ ) measured at $6 \mathrm{~K}$.

istically for unstrained bulk GaN. ${ }^{16}$ At low temperatures and for thin layers, strain-induced blue shift (up to $14 \mathrm{meV}$ ) of this peak has been reported for $\mathrm{GaN}$ grown on sapphire. ${ }^{14,15,17,18}$ Shan et al. ${ }^{17}$ showed that the amount of blue shift is proportional to the hydrostatic pressure on the sample, which induces additional strain in the epitaxial layer. From Fig. 2, the PL blue shift of our GaN grown on SOI (sample B) with respect to the strain-free $\mathrm{GaN}$ is only 4 $\mathrm{meV}$, which is considerably lower than the previously reported 10-14 meV for GaN grown on sapphire. This indicates clearly that growth of $\mathrm{GaN}$ on compliant SOI substrate reduces the strain in the epilayer by sharing it with the thin substrate.

Figure 3 shows the detailed UV emission spectrum of sample A of Fig. 1. Compared to sample B of Fig. 2 which was grown at $\sim 0.8 \mu \mathrm{m} / \mathrm{h}$ and $\mathrm{V} / \mathrm{III}=2500$, sample $\mathrm{A}$ has been grown with doubled growth rate $(\sim 1.5 \mu \mathrm{m} / \mathrm{h})$ and an increased V/III ratio of 3000 . Here, the neutral-donor bound exciton emission peak occurs at $3.471 \mathrm{eV}$. The electron mobility is improved from $178 \mathrm{~cm}^{2} / \mathrm{Vs}$ in sample $\mathrm{B}$ to $320 \mathrm{~cm}^{2} / \mathrm{Vs}$ in sample $\mathrm{A}$, respectively, for a constant low background carrier concentration of $3 \sim 4 \times 10^{17} \mathrm{~cm}^{-3}$.

In summary, GaN layers have been successfully grown on SOI substrates by LP-MOCVD. Crystal quality and electrical properties improved by surface nitridation increased bulk growth temperature and buffer growth rate and by reduction of the thickness of the top Si layer of the SOI substrate. XRD FWHM of the grown GaN was 366 arc sec, which is much better than for straight $\mathrm{GaN}$ growth on $\mathrm{Si}$. Electron mobility of $350 \mathrm{~cm}^{2} /(\mathrm{Vs})$ was achieved for a carrier concentration of $1.8 \times 10^{17} \mathrm{~cm}^{-3}$ indicating good crystal quality of the GaN on SOI. Photoluminescence measure- ments have been performed at both low temperature $(4 \mathrm{~K})$ and room temperature. At $6 \mathrm{~K}$, the PL of GaN is dominated by an intense and sharp near-band-gap UV emission around $3.47 \mathrm{eV}$, corresponding to the neutral-donor bound exciton. The FWHM of the peak can be as narrow as $18 \mathrm{meV}$. The yellow luminescence, which is characterized by a broad spectrum centered at $2.16 \mathrm{eV}$, is much weaker than the UV emission at this temperature. SIMS and XPS characterization suggest, that the yellow luminescence is associated with deep energy levels in the band gap, possibly due to $\mathrm{C}$ incorporation. The peak position of the UV emission changes both with temperature and strain. As the measurement temperature is increased to room temperature, the UV peak shows a red shift which corresponds well to the temperature dependence of the band gap. The strain-induced blue shift, compared to unstrained $\mathrm{GaN}$, of the $\mathrm{UV}$ emission at $6 \mathrm{~K}$ of our GaN layers grown on SOI is much less than for growth on sapphire, indicating strain relief by growth on the compliant SOI substrate.

The authors would like to thank M. Yoder and C. Wood for encouragement and support of this work. This work has been supported by ONR Contract No. N00014-92-J-1552 and by NSF/CNRS (INT-9217513).

${ }^{1}$ S. Nakamura, M. Senoh, S. Nagahama, N. Iwasa, T. Yamada, T. Matsushita, Y. Sugimoto, and H. Kiyoku, Appl. Phys. Lett. 70, 1417 (1997).

${ }^{2}$ S. C. Binari, K. Doverspike, G. Kelner, H. B. Dietrich, and A. E. Wickenden, Solid-State Electron. 41, 177 (1997).

${ }^{3}$ C. L. Chua, W. Y. Hsu, C. H. Lin, G. Christenson, and Y. H. Lo, Appl. Phys. Lett. 64, 3640 (1994).

${ }^{4}$ C. Carter-Coman, A. S. Brown, R. Bicknell-Tassius, N. M. Jokerst, and M. Allen, Appl. Phys. Lett. 69, 257 (1996).

${ }^{5}$ F. E. Ejeckam, Y. H. Lo, S. Subramanian, H. Q. Hou, and B. E. Hammons, Appl. Phys. Lett. 70, 1685 (1997).

${ }^{6}$ D. Pavlidis, J. Singh, Y. Park, A. Eisenbach, and J. Cao, Office of Naval Research Report (Contract No. N00014-92-J-1552), July 1996.

${ }^{7}$ J. Cao, D. Pavlidis, Y. Park, J. Singh, and A. Eisenbach, 8th Biennial Workshop on Organometallic Vapor Phase Epitaxy, April 13-17, 1997, Dana Point, California.

${ }^{8}$ A. R. Powell, S. S. Iyer, and F. K. LeGoues, Appl. Phys. Lett. 64, 1856 (1994).

${ }^{9}$ Z. Yang, F. Guarin, I. W. Tao, W. I. Wang, and S. S. Iyer, J. Vac. Sci. Technol. B 13, 789 (1995).

${ }^{10}$ A. J. Steckl, J. Devrajan, C. Tran, and R. A. Stall, J. Electron. Mater. 26, 217 (1997).

${ }^{11}$ D. Teng and Y. H. Lo, Appl. Phys. Lett. 62, 43 (1993).

${ }^{12}$ L. B. Freund and W. D. Nix, Appl. Phys. Lett. 69, 257 (1996).

${ }^{13}$ J. D. Colinge, Silicon-On-Insilator Technology: Materials to VLSI (Kluwer Academic, Norwell, Massachusetts, 1991).

${ }^{14}$ W. Grieshaber, E. F. Schubert, I. D. Goepfert, R. F. Karlicek, Jr., M. J. Schurman, and C. Tran, J. Appl. Phys. 80, 4615 (1996).

${ }^{15}$ D. A. Turnbull, X. Li, S. Q. Gu, E. E. Reuter, J. J. Coleman, and S. G. Bishop, J. Appl. Phys. 80, 4609 (1996).

${ }^{16}$ B. Monemar, Phys. Rev. B 10, 676 (1974).

${ }^{17}$ W. Shan, T. J. Schmidt, R. J. Hauenstein, and J. J. Song, Appl. Phys. Lett. 66, 3492 (1995).

${ }^{18}$ C. I. Harris, B. Monemar, H. Amano, and I. Akasaki, Appl. Phys. Lett. 67, 840 (1995). 